\title{
Enforcing periodic boundary conditions on general finite element discretisations of heterogeneous materials
}

\author{
A. I. Akpoyomare, M. I. Okereke \& M. S. Bingley \\ Department of Engineering Science, University of Greenwich, UK
}

\begin{abstract}
Predicting the effective thermo-mechanical response of heterogeneous materials such as composites, using virtual testing techniques, requires imposing periodic boundary conditions on geometric domains. However, classic methods of imposing periodic boundary conditions require identical finite element mesh constructions on corresponding regions of geometric domains. This type of mesh construction is infeasible for heterogeneous materials with complex architecture such as textile composites where arbitrary mesh constructions are commonplace. This paper discusses interpolation techniques for imposing periodic boundary conditions on general finite element mesh constructions for predicting the effective elastic properties of a variety of heterogeneous materials. Benchmark virtual tests on identical and non-identical meshes demonstrate the high fidelity of the proposed periodic boundary conditions enforcement technique, in comparison to a conventional technique of imposing periodic boundary conditions and experimental data.

Keywords: effective properties, periodic boundary condition, textile composite, mesoscale modelling, finite element, heterogeneous materials, virtual testing.
\end{abstract}

\section{Introduction}

Virtual tests can reduce the cost of experimental testing in the aerospace industry by $50 \%$ [1]. Furthermore, the predictive fidelity of virtual testing is determined chiefly by the accuracy of the geometric domain, material models and imposed boundary condition(s) (BC) [1]. For heterogeneous materials such as textile composites, periodic $\mathrm{BC}$ is the most efficient for virtual testing, with respect to accuracy, convergence rate and geometric domain size, in comparison to other common BCs such as Dirchlet and 
Neumann BCs $[1,2]$. However, imposing periodic BC on textile geometric domains is arduous because the classic implementation method requires homologous finite element (FE) meshes at the boundaries of a geometric domain. This homologous mesh requirement is difficult to satisfy for textile composites because of their complex geometric structure which yield inevitable non-homologous boundary mesh constructions [3]. Thus, it is desirable to develop techniques for imposing periodic BC on arbitrary mesh constructions amenable to textile composites.

Most recently, Jacques et al. [3] proposed a technique for imposing periodic BC to arbitrary textile meshes. Jacques and co-workers introduced several reference nodes in a Euclidean grid structure which were kinematically coupled to existing nodes on corresponding surfaces on the textile RVE. However, the use of Laplacian spatial averaging to determine the location of these reference nodes violates the strict enforcement of spatial 'homologousness' between boundary surface pairs, which is a pre-requisite for PCBs. Thus, numerical artefacts can ensue from this anomaly which may become apparent in finite deformation regimes. Tyrus et al. [4] imposed periodic BC to arbitrary unidirectional (UD) composite meshes in 2D using polynomial interpolation techniques. The displacement fields of fibres and matrix were interpolated using linear and cubic interpolants, respectively. Recently, Nguyen et al. [2] generalised the technique of Tyrus et al. [4] and extended the formalisms to 3D cases of UD and particulate composites. The authors used Lagrange and piecewise cubic Hermite polynomial interpolants to determine the displacement fields along independent boundary edges. Displacement fields on RVE surfaces were interpolated using a bi-linear Coons patch formulation.

In this communication, we describe and implement a dual-scale (micro and meso scale) homogenisation model for predicting the entire effective elastic properties of textile composites, using periodic BCs amenable to arbitrary textile meshes. We extend a robust variant of the periodic BC enforcement method proposed by Nguyen et al. [2]. Section 2 of this communication recalls the essentials of downscaling and periodic BC. Section 2.1 describes the proposed periodic BC technique amenable to arbitrary meshes. In Section 3, the proposed periodic BC method is validated. Lastly, Section 4 describes the adopted virtual testing technique used to determine the entire effective elastic properties of textile composites.

\section{Periodic boundary condition (PBC)}

To ensure energetic consistency between the macro-scale, $\Omega_{\text {macro }}$, and a sub-scale problem, $\Omega_{\mathrm{RVE}}$, boundary conditions on the boundary of the sub-scale, $\partial \Omega_{\mathrm{RVE}}$, must be specified as

$$
\oint_{\partial \Omega_{\mathrm{RVE}}}\left(t_{i}-\bar{\sigma}_{i j} n_{j}\right) u_{i} \mathrm{~d} S=0,
$$

where $\boldsymbol{t}$ is the external traction on $\partial \Omega_{\mathrm{RVE}}, \overline{\boldsymbol{\sigma}}$ is the volume-averaged stress in $\Omega_{\mathrm{RVE}}, \boldsymbol{n}$ is the outward normal to $\partial \Omega_{\mathrm{RVE}}$, and $\boldsymbol{u}$ is the imposed displacement field on $\partial \Omega_{\mathrm{RVE}}$.

Many BC's can be imposed on $\partial \Omega_{\mathrm{RVE}}$ to satisfy Eqn (1): however, periodic BC is the preferred $\mathrm{BC}$ of choice for heterogeneous materials. In order to impose PBC on $\Omega_{\mathrm{RVE}}$ in $\mathbb{R}^{N}$, where $N$ is the dimensionality of the RVE's solution space, $\partial \Omega_{\mathrm{RVE}}$ is 
decomposed into two distinct parts: a positive part, $\partial \Omega_{\mathrm{RVE}}^{+}$, and a negative part, $\partial \Omega_{\mathrm{RVE}}^{-}$. Each corresponding pair of positive and negative boundary faces have material points $\boldsymbol{x}^{+}$ and $\boldsymbol{x}^{-}$, respectively, such that, $\boldsymbol{x}^{+} \in \partial \Omega_{\mathrm{RVE}}^{+}$and $\boldsymbol{x}^{-} \in \partial \Omega_{\mathrm{RVE}}^{-} \cdot \boldsymbol{x}^{-}$and $\boldsymbol{x}^{+}$have unit outward normals, $\boldsymbol{n}^{+}=-\boldsymbol{n}^{-}$, respectively. Thus, the following relationships are satisfied

$$
\begin{array}{ll} 
& \partial \Omega_{\mathrm{RVE}}^{+} \cup \partial \Omega_{\mathrm{RVE}}^{-}=\partial \Omega_{\mathrm{RVE}}, \quad \text { and } \\
\left(\forall \boldsymbol{x}^{+} \in \partial \Omega_{\mathrm{RVE}}^{+} \text {and } \boldsymbol{x}^{-} \in \partial \Omega_{\mathrm{RVE}}^{-}\right) & \partial \Omega_{\mathrm{RVE}}^{+} \cap \partial \Omega_{\mathrm{RVE}}^{-}=\varnothing .
\end{array}
$$

In practice two different types of FE mesh constructions exist: (1) a homologous mesh construction, and (2) a non-homologous mesh construction. Homologous meshes satisfy specific conditions such that

$$
\begin{array}{ll}
\# \partial \Omega_{\mathrm{RVE}}^{+}=\# \partial \Omega_{\mathrm{RVE}}^{-} \quad \text { and } \\
\left(\forall \boldsymbol{x}^{+} \in \partial \Omega_{\mathrm{RVE}}^{+} \text {and } \boldsymbol{x}^{-} \in \partial \Omega_{\mathrm{RVE}}^{-}\right) & \boldsymbol{n}^{+} \times \boldsymbol{n}^{-}=\mathbf{0},
\end{array}
$$

where \# represents the cardinality of a set. Imposing PBC on homologous meshes is achieved by using classic methods that kinematically tie homologous boundary node pairs [1]. This kinematic tying is achieved using multi-point constraint equations. Conversely, non-homologous FE meshes satisfy specific conditions such that

$$
\begin{array}{ll}
\# \partial \Omega_{\mathrm{RVE}}^{+} \stackrel{?}{=} \# \partial \Omega_{\mathrm{RVE}}^{-}, \quad \text { and } \\
\left(\exists \boldsymbol{x}^{+} \in \partial \Omega_{\mathrm{RVE}}^{+} \text {and } \boldsymbol{x}^{-} \in \partial \Omega_{\mathrm{RVE}}^{-}\right) & \boldsymbol{n}^{+} \times \boldsymbol{n}^{-} \neq \mathbf{0} .
\end{array}
$$

The conditions described by Eqn (5) are illustrated in Fig. 1. In these cases, the classic kinematic tying of node pairs is unsuitable; therefore, more robust methods, proposed herein, must be utilised.

\subsection{Imposing PBC on arbitrary FE meshes}

In adopting the proposed $\mathrm{PBC}$ interpolation technique, we postulate that the displacement field of an RVE's boundary, $\partial \Omega_{\mathrm{RVE}}$, can be interpolated. Interpolation functions, $\mathbf{D}(\mathbf{s})$, are adopted such that Eqn (1) is satisfied. To this end, the following conditions are evoked to interpolate the displacement fields of the negative and positive parts of $\partial \Omega_{\mathrm{RVE}}$, respectively

$$
\begin{aligned}
& \boldsymbol{u}(\mathbf{s})^{-}=\mathbf{D}(\mathbf{s})=\sum_{k=1}^{n} \mathbf{N}_{k}(\mathbf{s}) \boldsymbol{a}_{k}, \quad \text { and } \\
& \boldsymbol{u}(\mathbf{s})^{+}=\mathbf{D}(\mathbf{s})+\boldsymbol{\varepsilon}\left(\boldsymbol{x}^{+}-\boldsymbol{x}^{-}\right),
\end{aligned}
$$

where $\mathbf{N}_{k}(\mathbf{s})$ for $k \in\{k=1,2, \cdots, n\}$ are shape functions which solely depend on spatial variable(s), s, $\boldsymbol{a}_{k}$ represents independent variables, $\varepsilon$ is the strain tensor imposed at the continuum scale, and $\left(\boldsymbol{x}^{+}-\boldsymbol{x}^{-}\right)$depends of the RVE's dimensions. Therefore the displacement field of $\partial \Omega_{\mathrm{RVE}}$, is determined from the independent variables $\boldsymbol{a}_{k}$ and the 


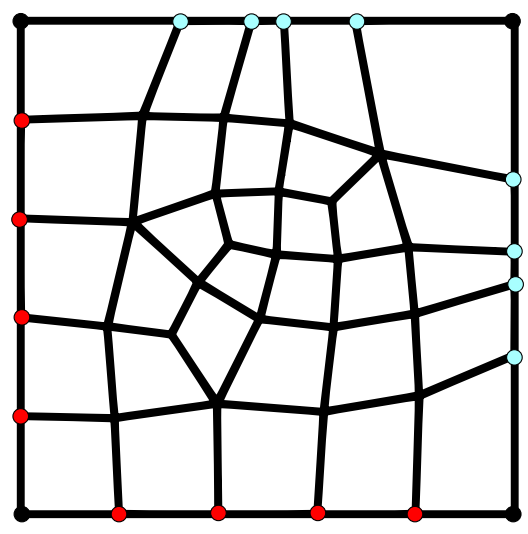

(a)

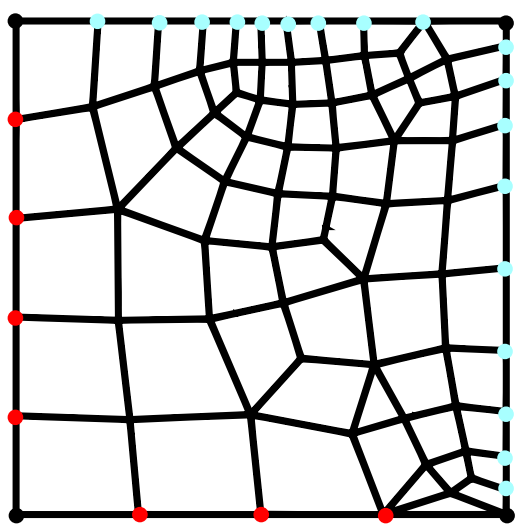

(b)

Figure 1: Typical examples of non-homologous FE meshes in 2D (a) Eqn (5a), and (b) Eqn (5b). The red and light blue circles $(\bullet,-)$ represents nodes on the -ve and +ve RVE boundaries, $\partial \Omega_{\mathrm{RVE}}^{-}$and $\partial \Omega_{\mathrm{RVE}}^{+}$, respectively. The black circles (a) represents vertex nodes which are shared by +ve and -ve RVE boundaries.

applied far-field continuum scale strain $\varepsilon$. The independent variables are selected as DOFs of specific nodes located at $\partial \Omega_{\mathrm{RVE}}^{-}$.

In $\mathbb{R}^{3}, \Omega_{\mathrm{RVE}}$ may be decomposed into edges and surfaces. Therefore, two different kinds of polynomial interpolants are necessary to interpolate the entire boundary of the RVE in $\mathbb{R}^{3}$ : an edge interpolant and a surface interpolant. A piecewise cubic Hermite spline and linear triangulation interpolation were adopted in this work for edge and surface interpolants because of their versatility. These interpolants are discussed in Section 2.1.1 and Section 2.1.2, respectively.

\subsubsection{Piecewise cubic Hermite interpolation for RVE edges}

To implement a piecewise cubic Hermite interpolant for an RVE's Edge, the edge is decomposed into $n$ segments $S_{i-1}$ for $i \in\{1,2, \cdots, n\}$ defined from $n+1$ triples $\left\{\left(\xi_{0}, \boldsymbol{u}_{0}, \boldsymbol{\theta}_{0}\right), \cdots,\left(\xi_{n}, \boldsymbol{u}_{n}, \boldsymbol{\theta}_{n}\right)\right\}$. Subsequently, the displacement field in each segment is interpolated using a third order Hermite polynomial:

$$
\begin{aligned}
& H_{1}(\zeta)=1-3 \zeta^{2}+2 \zeta^{3}, \\
& H_{2}(\zeta)=l\left(\zeta-2 \zeta^{2}+\zeta^{3}\right), \\
& H_{3}(\zeta)=3 \zeta^{2}-2 \zeta^{3}, \\
& H_{4}(\zeta)=l\left(-\zeta^{2}+\zeta^{3}\right),
\end{aligned}
$$

where $\zeta(\xi)=\frac{\xi-\xi_{i-1}}{l}, l=\xi_{i}-\xi_{i-1}$ and $\xi_{i-1} \leqslant \xi \leqslant \xi_{i}$. Thus, the displacement field in each segment is represented as

$$
\boldsymbol{u}(\xi)=H_{1}(\zeta(\xi)) \boldsymbol{u}_{i-1}+H_{2}(\zeta(\xi)) \boldsymbol{\theta}_{i-1}+H_{3}(\zeta(\xi)) \boldsymbol{u}_{i}+H_{4}(\zeta(\xi)) \boldsymbol{\theta}_{i},
$$


which can be written concisely in matrix form

$$
\boldsymbol{u}(\xi)=\tilde{\mathbf{N}} \tilde{\boldsymbol{q}},
$$

where $\tilde{\mathbf{N}}$ is the local shape function matrix for the interpolant, and $\tilde{\boldsymbol{q}}=$ $\left[\boldsymbol{u}_{i-1}^{T} \boldsymbol{\theta}_{i-1}^{T} \boldsymbol{u}_{i}^{T} \boldsymbol{\theta}_{i}^{T}\right]$ is the local vector of independent variables within each segment. Fig. 2 shows a schematic representation of implementing the this univariate interpolation technique for enforcing periodic BC to RVE edges.

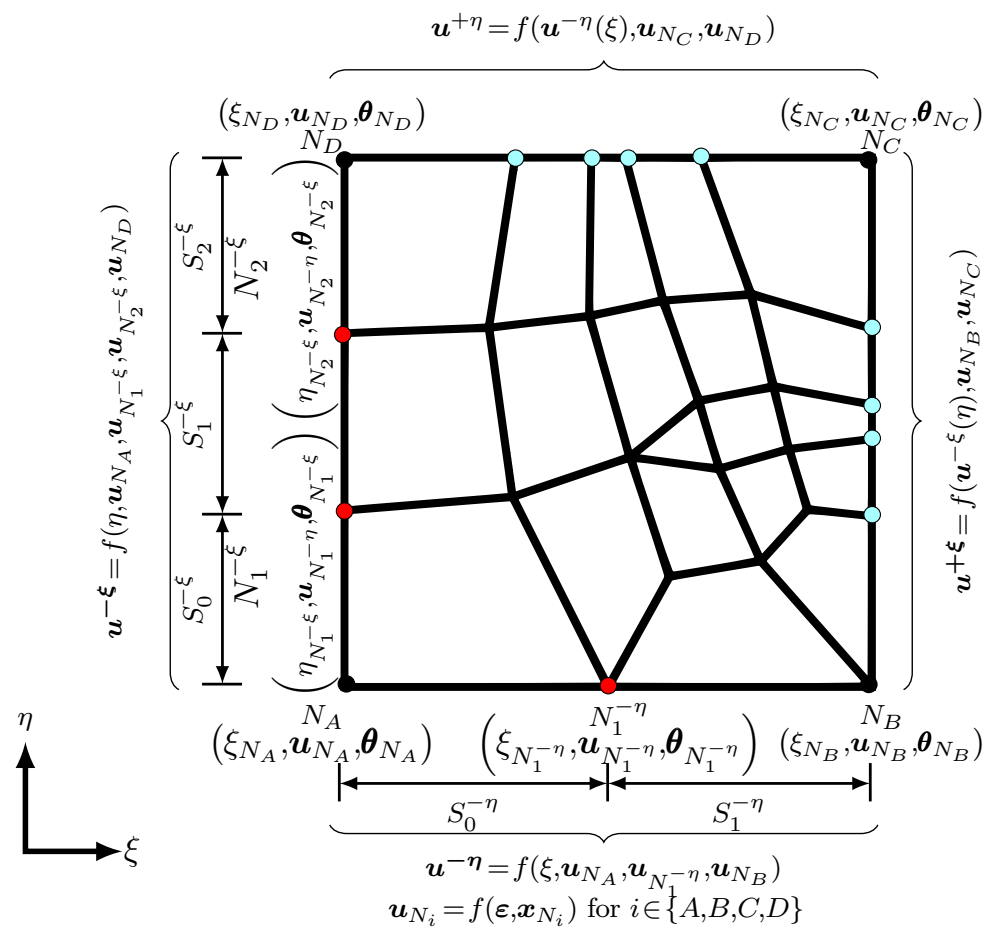

Figure 2: Schematic showing the implementation of PBC interpolation technique for RVE edges using univariate polynomial interpolation functions where $\boldsymbol{u}$ represents a displacement field, $\varepsilon$ represents the macroscopic strain tensor and $\boldsymbol{x}_{N_{i}}$ represents a material point of node $N_{i}$ for $i \in\{A, B, C, D\}$.

\subsubsection{Piecewise linear triangulation interpolation for RVE surfaces}

To implement a piecewise linear triangulation interpolant for an RVE's surface, the surface is decomposed into a collection of $n$ triangles $T_{i}$ for $i \in\{1,2, \cdots, n\}$ which define a triangulation $P$. The number of triangles, $n$, in the triangulation, $P$, is defined as $n=2 k-b-2$, where $k$ is the number of points in $P$ and $b$ is the number of points in $P$ that lie on the boundary of the convex hull of $P$. Many different triangulation 
techniques may be used to decompose the RVE's surface. This work adopts a Delaunay triangulation because it produces optimally shaped triangles which are necessary for good interpolation. Subsequently, the displacement field within each triangle, with vertices $v_{1}, v_{2}$ and $v_{3}$, is interpolated using a linear barycentric polynomial:

$$
\begin{aligned}
& B_{1}(\xi, \eta)=\frac{\operatorname{area}\left(v(\xi, \eta), v_{2}(\xi, \eta), v_{3}(\xi, \eta)\right)}{\operatorname{area}\left(v_{1}(\xi, \eta), v_{2}(\xi, \eta), v_{3}(\xi, \eta)\right)}, \\
& B_{2}(\xi, \eta)=\frac{\operatorname{area}\left(v(\xi, \eta), v_{1}(\xi, \eta), v_{3}(\xi, \eta)\right)}{\operatorname{area}\left(v_{1}(\xi, \eta), v_{2}(\xi, \eta), v_{3}(\xi, \eta)\right)}, \\
& B_{3}(\xi, \eta)=\frac{\operatorname{area}\left(v(\xi, \eta), v_{1}(\xi, \eta), v_{2}(\xi, \eta)\right)}{\operatorname{area}\left(v_{1}(\xi, \eta), v_{2}(\xi, \eta), v_{3}(\xi, \eta)\right)},
\end{aligned}
$$

where $B_{1}+B_{2}+B_{3}=1$. Thus, the displacement field within each triangle is represented as

$$
\boldsymbol{u}(\xi, \eta)=B_{1}(\xi, \eta) \boldsymbol{u}_{v_{1}}+B_{2}(\xi, \eta) \boldsymbol{u}_{v_{2}}+B_{3}(\xi, \eta) \boldsymbol{u}_{v_{3}},
$$

which can be written concisely in matrix form

$$
\boldsymbol{u}(\xi, \eta)=\tilde{\mathbf{N}} \tilde{\boldsymbol{q}},
$$

where $\tilde{\mathbf{N}}$ is the local shape function matrix for the interpolant, and $\tilde{\boldsymbol{q}}=\left[\boldsymbol{u}_{v_{1}} \boldsymbol{u}_{v_{2}} \boldsymbol{u}_{v_{3}}\right]$ is the local vector of independent variables within each triangle. Fig. 3 shows a schematic representation of enforcing periodic BC on RVE surfaces using piecewise linear triangulation interpolation functions.

\section{Validation of PBC enforcement for arbitrary FE meshes}

In validating the proposed method for periodic $\mathrm{BC}$ enforcement using polynomial interpolation, predictions using this method were compared to those obtained from PBC enforcement by kinematic tying [1] and experimental data. A unidirectional (UD) carbon fibrereinforced epoxy composite (T300/BSL914C) characterised in the world-wide failure exercise [5] was adopted due to the flexibility in controlling its FE mesh. The properties of the constituents comprising the T300/BSL914C composite are reported in Table 1. For the purpose of comparison with periodic BC enforcement by kinematic tying, a homologous RVE mesh was considered. However, for the case of a non-homologous mesh, only the proposed periodic $\mathrm{BC}$ enforcement using polynomial interpolation was amenable.

\subsection{Results and discussion of validation exercise}

Experimental data alongside predictions from the various PBC enforcement techniques are reported in Table 2. Furthermore, example computational FE contour plots from the different types of periodic BC enforcement techniques are depicted in Figs 4-5. For the homologous mesh, predictions from the periodic $\mathrm{BC}$ enforcement by kinematic tying and the proposed polynomial interpolation technique coalesce qualitatively and quantitatively. Coalescence is expected in this special case because for a homologous mesh where the entire nodes on the independent boundary regions are used as 


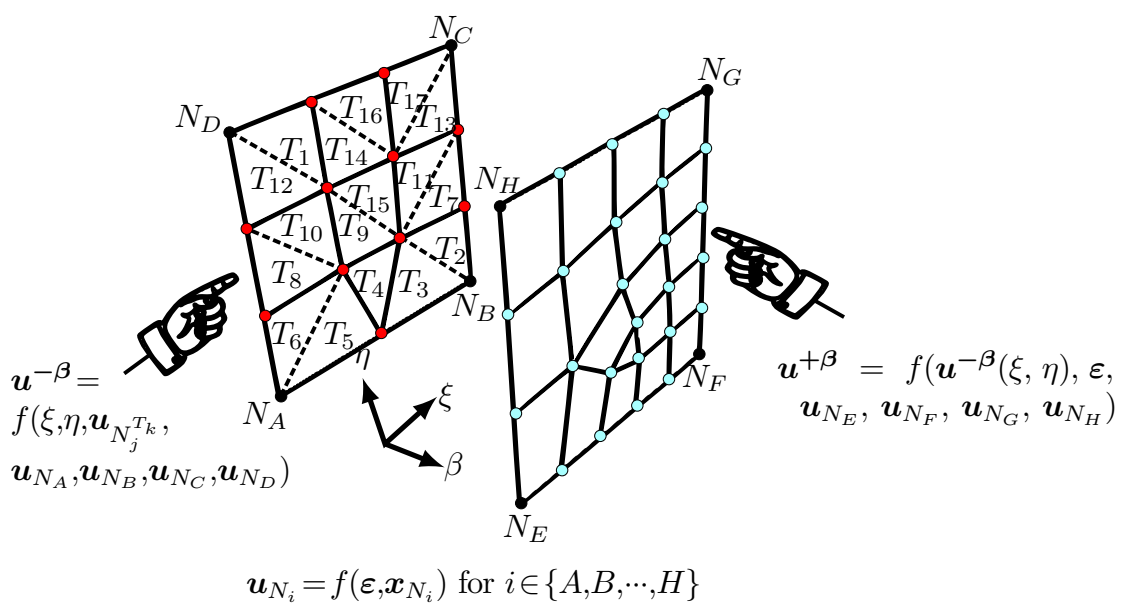

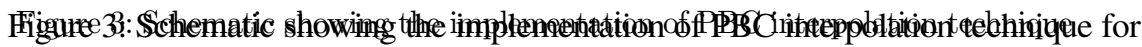

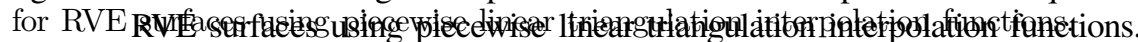

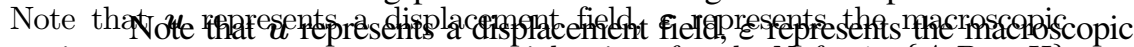

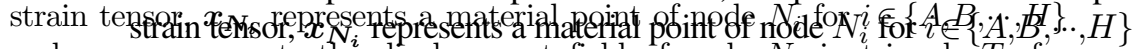

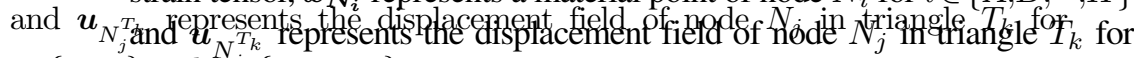
$i \in\{1,2,3\}$ and $k, 2,3\}$ and $\ddot{k} \in\{\}, 1,2, \cdots, 17\}$.

Table 1: Mechanical properties of the constituents of a carbon fibre-reinforced Table 1 : Mechanicał 858 Perties of the canstitueats of a carbon fibre-reinforced epoxy composite (T300/BSL914C) with $V_{f}=60 \%$ [亏ं].

\begin{tabular}{|c|c|c|}
\hline $\begin{array}{l}\text { Etastic constant } \\
\text { Elastic constant }\end{array}$ & \multirow{2}{*}{\multicolumn{2}{|c|}{$\begin{array}{l}\text { Fibre (T300) Matrix (BSL914C) } \\
\text { Fibre (T390) Matrix (BSL914C) } \\
230\end{array}$}} \\
\hline Longitudinal modulus (GPa), $E_{11}$ & & \\
\hline 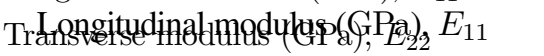 & 3.230 & $4^{4}$ \\
\hline 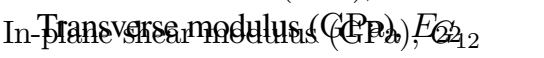 & 13.45 & 1.484 \\
\hline Transplane shear moduluss $\left(\left(G_{1} P_{a}\right)\right), G_{123}$ & 715 & 1.48481 \\
\hline MajyranBkeissorsheratimodulus (GPa), $G_{23}$ & 0.27 & 013481 \\
\hline Major Poisson's ratio, $\nu_{12}$ & 0.2 & 0.35 \\
\hline
\end{tabular}

contour plots from the different types of periodic BC enforcement techniques are depicted in Figs 4-5. For the homologous mesh, predictions from the periodic the independent degrees of freedom in the interpolation functions the interpolation method reduces to a kinematically tied case, Therefore, the kinematic tying periodic BC

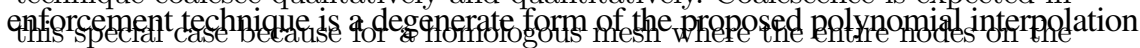

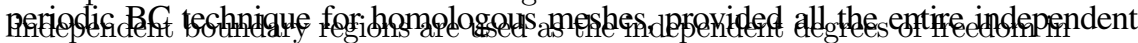

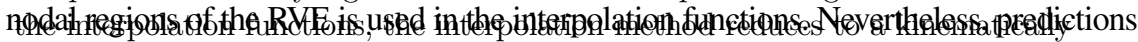

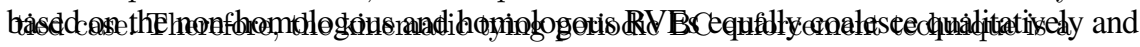
quantitatively. Although negligible differences are present within the FE contour plots, these differences stem from inevitable discretisation errors inherent within the RVEs due to mesh differences. Similarities between predictions from the homologous and 
non-homologous mesh is observed because the proposed PBC enforcement technique by polynomial implementation faithfully reproduces the appropriate boundary constraints on the RVE. More important, all the predicted effective elastic constants agree excellently with experimental data because appropriate boundary conditions have been enforced. Therefore, this virtual testing technique is well-suited for use in determining a holistic range of effective elastic constants of continuous fibre reinforced composites.

Table 2: Comparison of predicted effective elastic constants of T300/BSL914C ( $V_{f}=60 \%$ ) using different implementations of PBC and experimental data [5].

\begin{tabular}{|c|c|c|c|c|}
\hline \multirow[b]{2}{*}{$\begin{array}{l}\text { Elastic } \\
\text { constant }\end{array}$} & \multirow[b]{2}{*}{ Experiment } & \multicolumn{2}{|c|}{ Homologous mesh } & \multirow[b]{2}{*}{$\begin{array}{c}\text { Non-homologous mesh } \\
\text { PBC } \\
\text { enforcement } \\
\text { by } \\
\text { polynomial } \\
\text { interpolation }\end{array}$} \\
\hline & & $\begin{array}{c}\text { PBC } \\
\text { enforce- } \\
\text { ment by } \\
\text { kinematic } \\
\text { tying }\end{array}$ & $\begin{array}{c}\text { PBC } \\
\text { enforcement by } \\
\text { polynomial } \\
\text { interpolation }\end{array}$ & \\
\hline$E_{11}(\mathrm{GPa})$ & 138 & 133 & 133 & 133 \\
\hline$E_{22}(\mathrm{GPa})$ & 11 & 10 & 10 & 10 \\
\hline$E_{33}(\mathrm{GPa})$ & $11^{*}$ & 10 & 10 & 10 \\
\hline$\nu_{12}$ & 0.28 & 0.23 & 0.23 & 0.23 \\
\hline$\nu_{13}$ & $0.28^{*}$ & 0.23 & 0.23 & 0.23 \\
\hline$\nu_{23}$ & 0.4 & 0.37 & 0.37 & 0.37 \\
\hline$G_{12}(\mathrm{GPa})$ & 5.5 & 4.2 & 4.2 & 4.2 \\
\hline$G_{13}(\mathrm{GPa})$ & $5.5^{*}$ & 4.2 & 4.2 & 4.2 \\
\hline$G_{23}(\mathrm{GPa})$ & $3.9^{* *}$ & 3.3 & 3.3 & 3.3 \\
\hline
\end{tabular}

* Transverse isotropy in the 2-3 plane is assumed.

** Computed based on transverse isotropy in the 2-3 plane by $G_{23}=\frac{E_{22}}{2\left(1+\nu_{23}\right)}$.

\section{Predicting the effective elastic properties of textile composites with arbitrary FE mesh}

Having validated the proposed PBC enforcement technique for arbitrary FE meshes, using a UD composite as a test case, the next step is to use this validated technique to predict the effective elastic properties of textile composites. The selected test material for the analysis is a through-thickness angle-interlock epoxy composite (TTT-AIC) with low crimp [6]. The idealised geometry of the TTT-AIC is depicted in Fig. 6. The entire yarns within the composite comprise carbon fibre rovings: the warp and weft yarns comprise Tenax HTS whilst the binder yarns comprise Tenax HTA. The composite was 


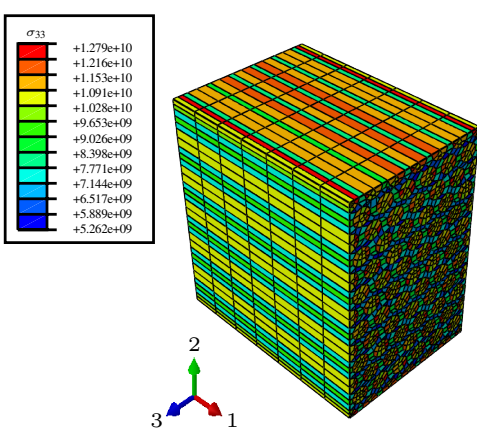

(a)

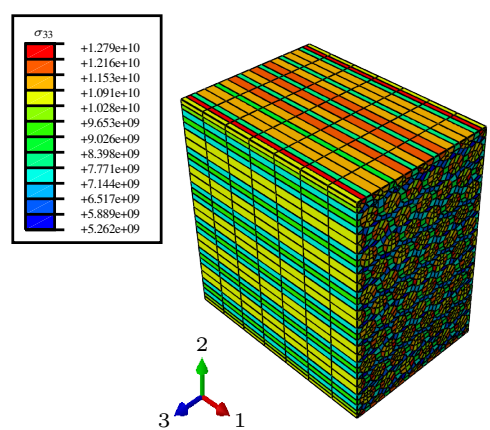

(b)

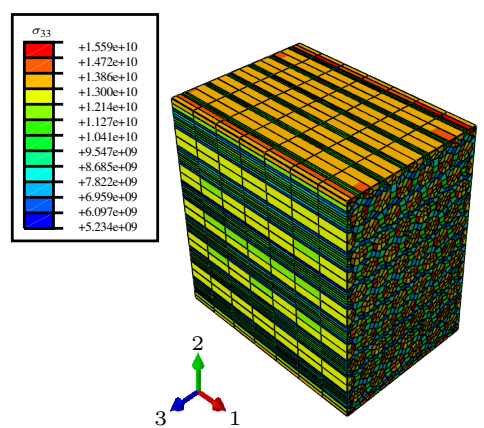

(c)

Figure 4: Comparison of FE contour plots illustrating longitudinal tensile deformation (i.e. $\varepsilon_{33}$ ) of T300/BSL914C (a) homologous mesh with PBC enforcement by kinematic tying, (b) homologous mesh with $\mathrm{PBC}$ enforcement by polynomial interpolation, and (c) non-homologous mesh with PBC enforcement by polynomial interpolation.

consolidated used an RTM-6 epoxy resin. The material properties of the constituents comprising this textile are reported in Table 3.

An in-house textile composite generating algorithm, TextCompGen, was developed and implemented in ABAQUS/CAE to generate textile RVEs.

In analysing the textile composite the yarns were decomposed into their primary constituents: matrix and fibre. Thereafter, yarns were modelled at the micro-scale as, densely packed, orthotropic UD composites (i.e. an identical analysis technique was used in Section 3). Subsequently, the homogenised effective elastic constants extracted from the micro-scale analysis were used as inputs for the meso-scale continuum model for each yarn. The arbitrary undulation of each yarn was considered by assigning discrete material orientations to each yarn within the fabric. Furthermore, the discrete matrix pockets at the meso-scale are modelled using an identical Hookean elastic model used in the micro-scale analysis. 


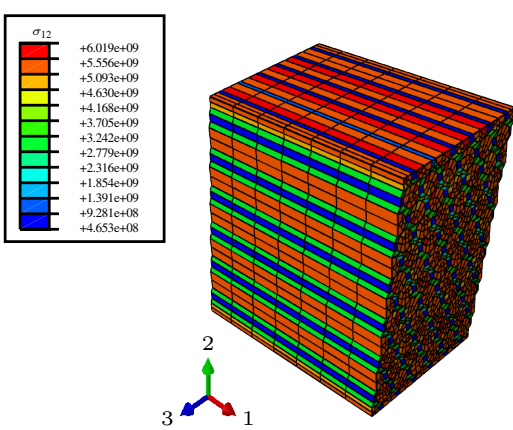

(a)

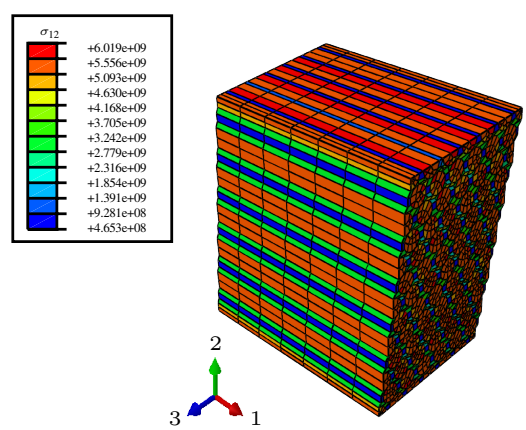

(b)
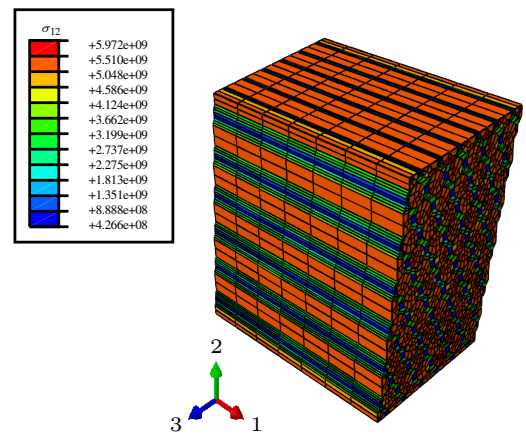

(c)

Figure 5: Comparison of FE contour plots illustrating in-plane shear deformation (i.e. $\varepsilon_{12}$ ) of T300/BSL914C (a) homologous mesh with PBC enforcement by kinematic tying, (b) homologous mesh with $\mathrm{PBC}$ enforcement by polynomial interpolation, and (c) non-homologous mesh with PBC enforcement by polynomial interpolation.

Table 3: Mechanical properties of the constituents of the through-the-thickness angle-interlock textile composite [6].

\begin{tabular}{lccc}
\hline Elastic constant & Tenax HTA & Tenax HTS & Epoxy \\
\hline Longitudinal modulus (GPa), $E_{11}$ & 240 & 240 & 2.84 \\
Transverse modulus (GPa), $E_{22}$ & 14 & 14 & 2.84 \\
In-plane shear modulus (GPa), $G_{12}$ & 20 & 20 & 1.029 \\
Transverse shear modulus $(\mathrm{GPa}), G_{23}$ & 10 & 10 & 1.029 \\
Major Poisson's ratio, $\nu_{12}$ & 0.3 & 0.3 & 0.38 \\
Minor Poisson's ratio, $\nu_{23}$ & 0.39 & 0.39 & 0.38 \\
\hline
\end{tabular}




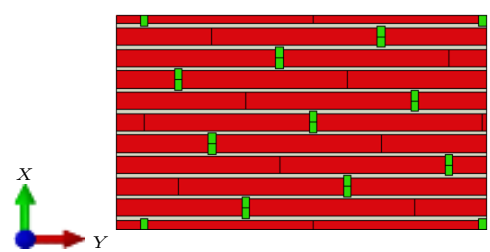

(a)

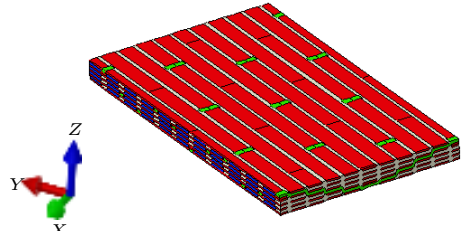

(b)

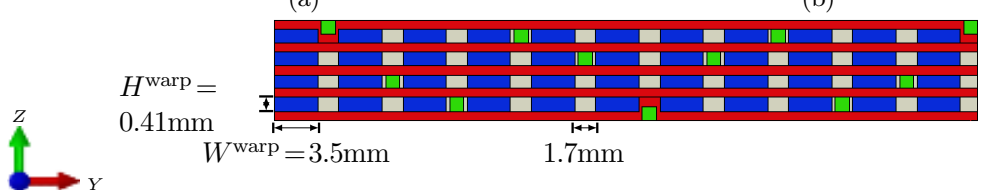

(c)

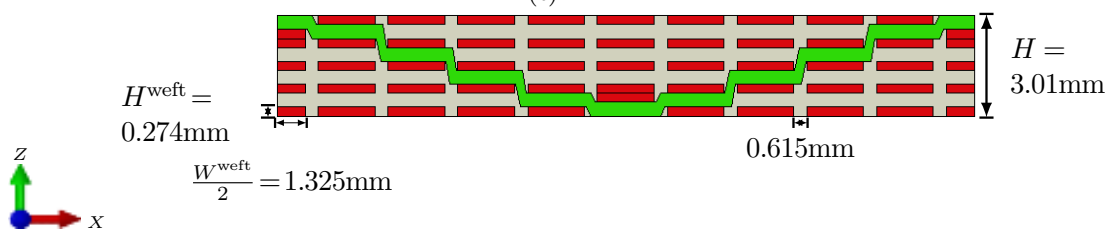

(d)

Figure 6: Idealised geometric model of the TTT-AIC fabric: (a) XY plane view,

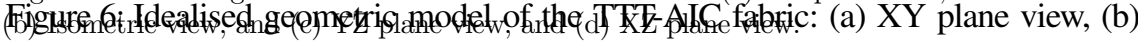
Isometric view, and (c) YZ plane view, and (d) XZ plane view.

In analysing the textile composite the yarns were decomposed into their primary constituents: matrix and fibre. Thereafter, yarns were modelled at the 4.1.Results, and discussion, nothopic UD composites (i.e. an identical analysis technique was used in Section 3). Subsequently, the homogenised effective elas-

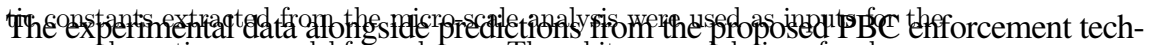

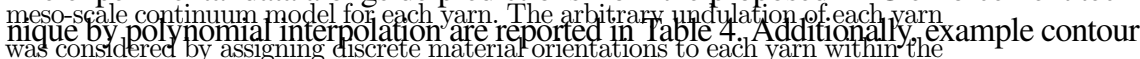

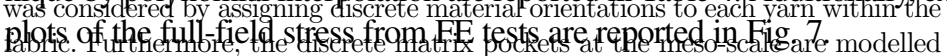

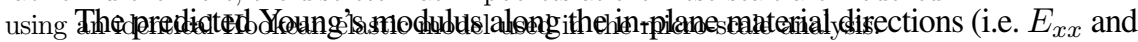
$\left.E_{y y}\right)$ from the virtual test corroborates experimental results. However, the virtual test milargiesalty andrDisedisitsnthe stiffness in both cases with a predictive discrepancy of about $2 \%$. This over-prediction may be borne from the regularity of the virtual model in com-

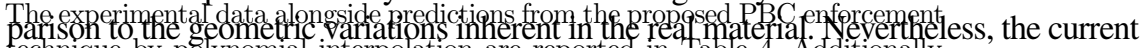

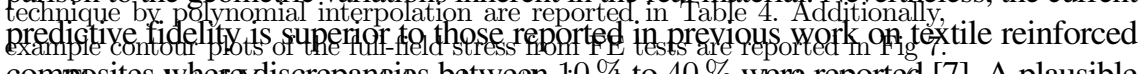

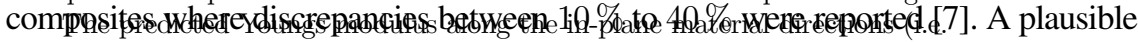

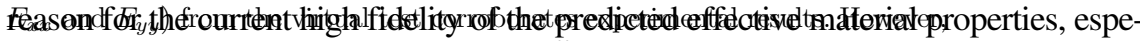

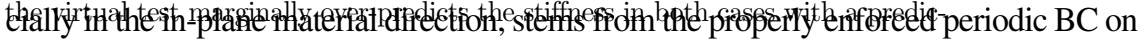
the textile domain as well as the dual-scale homogenisation strategy adopted in this work.

In comparison to experiments, the current virtual test over-predicts the throughthe-thickness Young's modulus (i.e. $E_{z z}$ ), by about $36 \%$. This discrepancy is in line with predictions from previous work on similar through-the-thickness reinforced fabric [7]. It is noted, however, that the experimental value for $E_{z z}$ was inferred from a through-thickness compression test which introduces inherent experimental uncertainties of $15 \%$ [7]. Furthermore, the overestimation of $E_{z z}$ by the virtual test most likely stems 


$\begin{array}{lcc}\nu_{y z} & - & 0.380 \\ G_{x y}(\mathrm{GPa}) & 11 & 2.98\end{array}$

140 High Performance and Optimum Design of Structures and Materials II $G_{y z}(\mathrm{GPa})$ 3.17

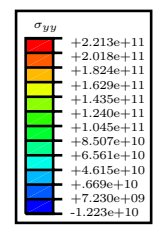

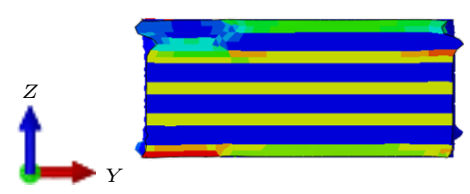

(a)

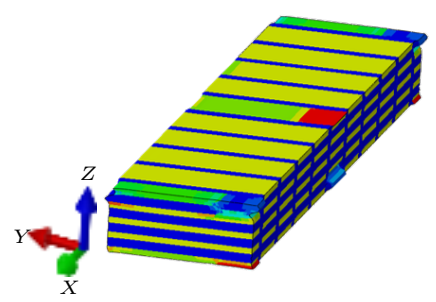

(b)

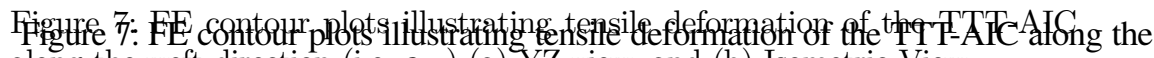

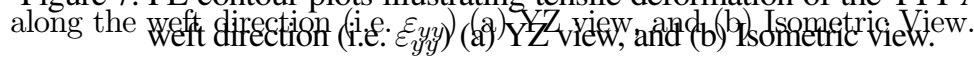

\section{Conclusions}

A virtual testing framework for characterising the mechanical response of typical heterogeneous materials with a robust technique for enforcing periodic bound-

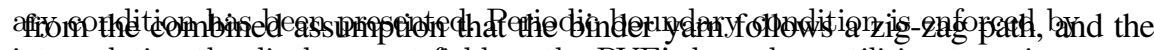

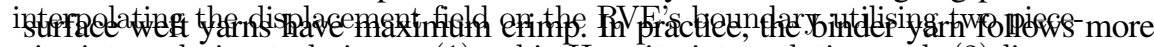

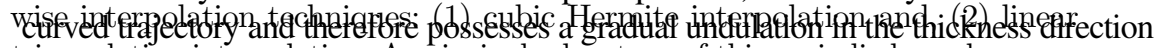

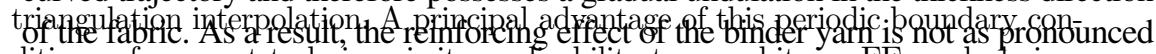

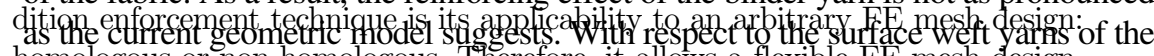

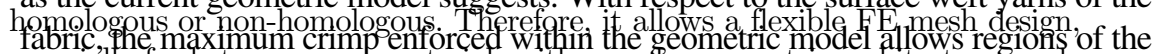
especially for heterogeneout materials with comptex geometric architectures such

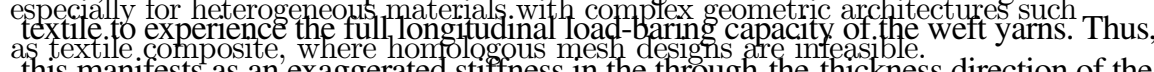

this manifests as an' exaggerated stiffness in the throughthe-thickness direction of the

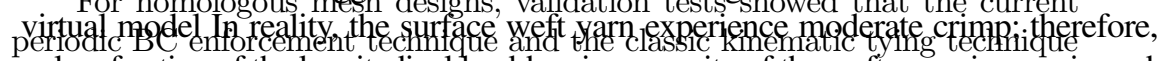

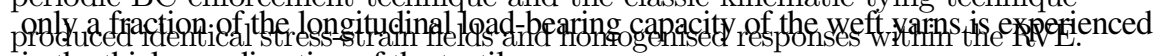
in the thickness direction of the textile.

There is a $73 \%$ discrepancy between the predicted in-plane shear modulus (i.e. $G_{x y}$ ) and the reported experimental data. The principal source of this discrepancy is most likely from the experimental data reported by the originating authors [6]. The authors performed a $45^{\circ}$ off-axis tensile test on the TTT-AIC specimen; however, the mandatory data reduction steps necessary for this test method was not reported in their work, casting doubt on its veracity. Previous work on experimental determination of in-plane shear modulus [8] of a similar TTT-AIC fabric reported average values of about $4 \mathrm{GPa}$. Furthermore, previous work on experimental and virtual characterisation of woven textile composites reported that the shear moduli of these composites are similar to those of its constituent yarns. Hence, results from the current work is qualitatively, and to a large degree, quantitatively consistent with these previous findings on the in-plane shear modulus, (i.e. $G_{x y}$ ), of the composite. The predicted magnitudes of the through-thickness shear moduli (i.e. $G_{x z}$ and $G_{y z}$ ) are equally similar to that of the in-plane shear modulus (i.e. $G_{x y}$ ) of the composite.

More important, the originating authors [6] did not report experimental data for the Poisson's ratio of the composite. However, the current virtual test predicted the entire Poisson's ratios and the reported magnitudes are qualitatively similar to those reported for a comparable woven textile composites [9]. 
Table 4: Comparison of predicted elastic constants of the TTT-AIC textile using the proposed PBC enforcement technique and experimental data [6].

\begin{tabular}{lcc}
\hline Elastic constants & Experiment & Virtual test \\
\hline$E_{x x}(\mathrm{GPa})$ & 64 & 65.4 \\
$E_{y y}(\mathrm{GPa})$ & 62 & 62.6 \\
$E_{z z}(\mathrm{GPa})$ & 7 & 9.5 \\
$\nu_{x y}$ & - & 0.045 \\
$\nu_{x z}$ & - & 0.370 \\
$\nu_{y z}$ & - & 0.380 \\
$G_{x y}(\mathrm{GPa})$ & 11 & 2.98 \\
$G_{x z}(\mathrm{GPa})$ & - & 3.01 \\
$G_{y z}(\mathrm{GPa})$ & - & 3.17 \\
\hline
\end{tabular}

\section{Conclusions}

A virtual testing framework for characterising the mechanical response of typical heterogeneous materials with a robust technique for enforcing periodic boundary condition has been presented. Periodic boundary condition is enforced by interpolating the displacement field on the RVE's boundary utilising two piecewise interpolation techniques: (1) cubic Hermite interpolation and, (2) linear triangulation interpolation. A principal advantage of this periodic boundary condition enforcement technique is its applicability to an arbitrary FE mesh design: homologous or non-homologous. Therefore, it allows a flexible FE mesh design, especially for heterogeneous materials with complex geometric architectures such as textile composite, where homologous mesh designs are infeasible.

For homologous mesh designs, validation tests showed that the current periodic $\mathrm{BC}$ enforcement technique and the classic kinematic tying technique produced identical stress-strain fields and homogenised responses within the RVE. This demonstrated that in this special mesh design case, the kinematic tying method is a degenerate form of the periodic $\mathrm{BC}$ enforcement by interpolation. Whereas for the non-homologous mesh design, only the periodic $\mathrm{BC}$ enforcement by interpolation was applicable. For this non-homologous mesh case, results showed that the interpolation technique produced similar stress-strain fields and homogenised responses within the RVE in comparison to the homologous mesh case, barring negligible FE discretisation errors. Consequently, the interpolation technique is a superior technique for enforcing periodic boundary conditions because of its additional applicability to non-homologous meshes.

\section{References}

[1] Okereke, M.I. \& Akpoyomare, A.I., A virtual framework for prediction of full-field elastic response of unidirectional composites. Computational Materials Science, 70(null), pp. 82-99, 2013. 
[2] Nguyen, V.D., Béchet, E., Geuzaine, C. \& Noels, L., Imposing periodic boundary condition on arbitrary meshes by polynomial interpolation. Computational Materials Science, 55, pp. 390-406, 2012.

[3] Jacques, S., De Baere, I. \& Van Paepegem, W., Application of periodic boundary conditions on multiple part finite element meshes for the meso-scale homogenization of textile fabric composites. Composites Science and Technology, 92, pp. 41-54, 2014.

[4] Tyrus, J.M., Gosz, M. \& DeSantiago, E., A local finite element implementation for imposing periodic boundary conditions on composite micromechanical models. International Journal of Solids and Structures, 44(9), pp. 2972-2989, 2007.

[5] Hinton, M.J., Kaddour, A.S. \& Soden, P.D., Failure Criteria in Fibre Reinforced Polymer Composites: The World-Wide Failure Exercise. Elsevier, p. 1255, 2006.

[6] Gerlach, R., Siviour, C.R., Wiegand, J. \& Petrinic, N., In-plane and throughthickness properties, failure modes, damage and delamination in 3D woven carbon fibre composites subjected to impact loading. Composites Science and Technology, 72(3), pp. 397-411, 2012.

[7] Buchanan, S., Grigorash, A., Archer, E., McIlhagger, A., Quinn, J. \& Stewart, G., Analytical elastic stiffness model for 3D woven orthogonal interlock composites. Composites Science and Technology, 70(11), pp. 1597-1604, 2010.

[8] Buchanan, S., Archer, E., Townsend, D., Jenkins, S., McIlhagger, A. \& Quinn, J., Determination of in-plane shear modulus of 3D woven composites with large repeat unit cells. Plastics, Rubber and Composites, 41(4), pp. 194-198, 2012.

[9] Cox, B.N. \& Dadkhah, M.S., The Macroscopic Elasticity of 3D Woven Composites. Journal of Composite Materials, 29(6), pp. 785-819, 1995. 